\title{
ESTRATEGIAS DEL RELATO DE VIAJES EN JUAN RUIZ Y EL MARQUÉS DE SANTILLANA
}

\author{
Miguel Ángel Pérez Priego \\ Universidad Nacional de Educación a Distancia - UNED \\ mperez@flog.uned.es
}

$\mathrm{E}$ n el comienzo de todo estaba el camino. A pesar de su vida aparentemente sedentaria y la precariedad de los medios de transporte, el hombre medieval fue de una gran actividad viajera. En el viaje transcurría buena parte de la vida del rey, del clérigo o del caballero. Y la ruta era el punto de encuentro, tanto en la sincronía horizontal del momento como en la vertical de la historia. El camino, construido muchas veces sobre antiguas calzadas romanas, estaba jalonado de castillos, monasterios, santuarios, posadas; surcaba valles, sierras y llanadas, y por él transitaban todo tipo de gentes, desde héroes de los cantares de gesta y devotos peregrinos a astutos mercaderes, clérigos vagantes y caballeros de fortuna.

En el entrelazado de caminos, calzadas y veredas que cruzaban las tierras de la cuenca alta del río Manzanares, al norte de la provincia de Madrid, fue muy concurrida la vía que iba de Alcalá y Guadalajara hasta Colmenar y Segovia, atravesando terrenos de las actuales Torrelaguna, Tres Cantos y Manzanares. Ese camino unió en el tiempo a dos de los más grandes poetas medievales, que lo recorrieron en ocasiones diferentes: el enamoradizo trotamundos Juan Ruiz, arcipreste de Hita, y el culto y poderoso caballero, también cautivo de amores, don Íñigo López de Mendoza, marqués de Santillana.

Juan Ruiz recorrió esa vía cuando, en la cuaresma de uno de los años de mediados del siglo XIV, fue a «probar la sierra» y emprendió ruta hacia los parajes de Guadarrama y el puerto de la Fuenfría, donde se encontró con las cuatro serranas salteadoras, bravas y desafiantes. Íñigo López, un siglo después, heredó gran parte de aquellas tierras en mayorazgo (Manzanares y Buitrago de Lozoya) y no serían infrecuentes sus viajes desde el palacio de Guadalajara para cuidarlas y defenderlas. También cantaría sus encuentros con las serranas del valle de la Gamella y el Boalo y, un poco más al norte, con las de Lozoyuela y Buitrago.

Ese recorrido, en efecto, llevó a Juan Ruiz a internarse por aquellos lugares serranos, desde el valle de Lozoya y el puerto de Malangosto a Sotosalbos y Segovia, de donde regresará por el puerto de la Fuenfría. Deambulando por esos parajes se producen los encuentros sucesivos con las cuatro serranas: la Chata de Malangosto, Gadea de Riofrío, Menga la serrana del Cornejo y Alda de Tablada

Edad de Oro, XL (2021), pp. 51-62, ISSN: 0212-0429 - ISSNe: 2605-3314

DOI: https://doi.org/10.15366/edadoro2021.40.002 
(Tate, 1970; Kirby, 1976; Di Stefano, 2001). Tras estas aventuras, se retirará a hacer oración en el cercano santuario de Santa María del Vado.

Tal es el breve recorrido geográfico de Juan Ruiz, que - a pesar de su condensación narrativa y su subordinación al argumento principal del Libro de buen amor - se nos presenta como un auténtico relato de viajes. Hay, lo primero, una motivación y categorización del viaje como tal. Este no es otra cosa que una peregrinación, una peregrinación íntima, si se quiere, en la que, tras la peripecia, se busca el perdón y la confortación del alma. El arcipreste emprende esa peregrinación después de sus fracasos amorosos y tras pedir perdón a las dueñas, y la termina, luego de sus aventuras serranas, retirándose a Santa María del Vado, al pie de la sierra, a «tener í vigilia, como es acostunbrado» $(1.044 c)^{1}$, pues en siete días se acerca el tiempo de Cuaresma, conforme nos recuerda tras orar a la Virgen y meditar sobre la Pasión de Cristo («dende a siete días era Quaresma tanto», $1.067 \mathrm{c})$.

En segundo lugar, hay en el breve relato un atento cuidado de su presentación en el marco espacial y temporal. En efecto, el itinerario está perfectamente marcado y transitado por el viajero. Procedente de tierras alcarreñas, recorre el camino serrano, cuyo primer hito mencionado es Malangosto, que le lleva a Segovia, de allí emprende camino de retorno por la Fuenfría, llega a Riofrío y a Otero de los Herreros, luego a la llamada venta Cornejo, camino del puerto de la Tablada, del que desciende de madrugada, y termina retirándose a la ermita de Santa María del Vado en las proximidades (Calleja Guijarro, 2004).

Dicho con más detalle, el arcipreste decide internarse en la sierra de Lozoya, cuando corría el día 3 marzo: «El mes era de março, día de sant Meder (Emeterio)» (c. 951a); tras el encuentro con la Chata de Malangosto, estuvo tres días («Torné para mi casa luego al tercer día», c. 974a), parece que no muy felices, en Segovia (la de «la serpiente groya», donde no halló «pozo dulce nin fuente perhenal», 972c-973b). Emprende camino de vuelta por el puerto de la Fuenfrida y tiene el encuentro con Gadea de Riofrío, con quien comparte el almuerzo, pero no pasa toda la tarde, pues todavía «con sol temprano» (c. 985d) llega a la aldea de Otero de los Herreros. El «lunes antes del alva» (c. 993a) emprende camino hacia el puerto de la Tablada y cerca de Cornejo tiene el encuentro con la amable y comedida Menga Lloriente, y recuerda que pasó de mañana el puerto («pasé de mañana el puerto por sosegar tenprano», c. 996d); a la carrera desciende cuesta abajo («corrí la cuesta ayuso», c. 1.007b) y al pie del puerto, de madrugada («fálleme con Alda / a la madrugada», c. 1.022cd) se encuentra con la trefuda Alda de Tablada, que no le dará hospedaje. A continuación, se retira a Santa María del Vado, a la bajada de la sierra.

Todas las citas del texto se hacen por la edición de Alberto Blecua (1992). 
Todo ocurre con mucha celeridad, muy rápido, prácticamente en una semana. Calculemos: del 3 de marzo al lunes (que son las dos referencias temporales mencionadas expresamente: «día de sant Meder» y «lunes antes del alva») han debido de pasar - descontando hacia atrás - un domingo (en compañía de Gadea y llegada a Otero), tres días antes está en Segovia (sábado, viernes, jueves), el miércoles se halla quizá de camino, el 3, que sale a la sierra y sería el martes, y pasa esa noche con la Chata en Malangosto. El lunes expresamente mencionado es el día siguiente al que estuvo con Gadea y hubo de pasarlo con Menga, el martes (un nuevo martes) de mañana se encuentra con Alda y ese mismo día tiene la vigilia en Santa María del Vado. Al día siguiente, miércoles según nuestros cálculos, nos recuerda que dentro de siete días se inicia la cuaresma, es decir, es el miércoles de ceniza. El arcipreste ha pasado las cuatro aventuras de la sierra siete días antes de la cuaresma, como una especie de preparación especial, pero también de desmesura, de desbordamiento de la línea sentimental del libro (Riquer, 1969).

Aparte del itinerario y el orden cronológico, hay otras estrategias del discurso viajero también desplegadas por Juan Ruiz. Una de ellas, bien significativa, es la que tiene que ver con el descubrimiento del otro, en este caso representado por las cuatro serranas. En ese viaje de peregrinación amatoria y purgación íntima que se plantea, es fundamental la ampliación de encuentros para completar el conocimiento de todas las gentes, para probar todas las cosas, como manda el apóstol. Eso es lo que motiva el encuentro sucesivo con las cuatro serranas salteadoras, la Chata, Gadea, Menga y Alda. En ellas descubre el arcipreste a sus otras amantes, distintas y sorprendentes, que amplían la perspectiva de las que venía conociendo y tratando en el libro, más conformes con los patrones convencionales femeninos. Las serranas son personajes distintos, extremados en su apariencia, en sus gestos y compostura. Son las serranas guiadoras y salteadores de la tradición popular, cuya descripción y comportamiento ha acentuado y deformado el autor.

El descubrimiento y sorpresa es, tal vez, lo que justifica aquí el recurso a la parodia como estrategia de persuasión (Deyermond, 1970; Gernet, 2009; Simonatti, 2008). La logra el autor mediante la modificación de los elementos esenciales de la pastorela con motivos amplificados o deformados de los cantares de serrana: el protagonista, en lugar del caballero galante, es ahora la figura poco apuesta del clérigo a lomos de una mula; el mal tiempo invernal de nieve y granizo ha desplazado a la bonanza de la primavera, el escenario arriscado del puerto ha sustituido al locus amœenus; y, en fin, la serrana salteadora, hombruna, bravía y deforme ha ocupado el lugar de la pastora idealizada. Esta pastora idealizada y casi siempre recatada de las pastorelas, aquí ha sido sustituida por el tipo de serrana salteadora de la tradición folclórica. Pero además, Juan Ruiz ha subrayado de forma más extrema sus rasgos: ha acrecentado su carácter hombruno y bravío, resaltando su 
fealdad, su destreza en el manejo del cayado y la honda o su actitud desafiante ante el viajero: la Chata de Malangosto, «gaha, roín e heda», 961b; «arrojóme la cayada / e rodeóme la fonda, / enaventóme el pedrero, / diz: “¡Par el Padre verdadero, / tú me pagarás oy la ronda!”», 963; Gadea, «la chata maldita, / diome con la cayada tras la oreja, fita. / Derribome cuesta ayuso e caí estordido», 977cd y 978a; la del Cornejo le exige casamiento y una larga lista de presentes («coidós cassar conmigo, como con su vezino», 993d); Alda, al pie del puerto, es un auténtico vestiglo, «la más grande fantasma que vi en este siglo, / yeguariza trefuda, talla de mal ceñiglo», $1.007 \mathrm{~cd}$.

A esas notas ha añadido aun un crudo erotismo, que hace que sea ahora la serrana quien, más que requerir de amores, fuerce al amante viajero a la lucha venérea. Con la Chata y Gadea se ve obligado a mantener contacto sexual («Por la muñeca me priso, / ove de fazer quanto quiso: / creet que fiz buen barato», 971efg; «Tomome por la mano e fuémosnos en uno... / díxome que jugásemos el juego por mal de uno», 981ad); se libra, en cambio, de Alda (le da posada en la Tablada pero no tiene moneda para pagar el hostalaje); y parece que escapa de las bodas con Menga Lloriente prometiendo volver con los regalos («luego fagamos las bodas... / que ya vo por lo que pides», 1.005d-f).

El retrato físico de la serrana, que introduce Juan Ruiz con Alda de Tablada (cs. 1.010-1.020) es también una inversión paródica, del canon convencional de belleza femenina de entonces. Como se puede leer en el libro de la doncella Teodor, ese canon convencional venía a estar fijado en dieciocho rasgos, agrupados en seis categorías de tres cada una:

Digo que luenga en tres, que sea luenga d'estado e que haya el cuello largo e los dedos luengos; e blanca en tres: el cuerpo blanco e los dientes blancos e lo blanco de los ojos blanco; e prieta en tres: cabellos prietos e las cejas prietas e lo prieto de los ojos prieto, e bermeja en tres: labros, mexillas, enzías; e pequeña en tres: boca pequeña, naris pequeña e los pies pequeños; e ancha en tres: ancha de caderas e ancha de espaldas e ancha la fruente (Knust, 1879: 514).

A él se ajusta más o menos Juan Ruiz cuando hace, por ejemplo, el retrato de la dueña encerrada (cs. 431-435): mujer de talla, de cabeza pequeña, cabellos rubios, cejas apartadas, ojos grandes y relucientes, orejas pequeñas, cuello alto, nariz afilada, dientes menudillos y blancos, labios bermejos, boca pequeña, tez blanca, clara y lisa, ancheta de caderas. Con esta belleza convencional, ajustada al canon y a la tradición retórica y literaria, contrasta el retrato de Alda de Tablada, cuyos rasgos negativos son todo un contrapunto de los rasgos positivos de la descriptio puellae: 
Avía la cabeça mucho grande, sin guisa, cabellos chicos, negros, más que corneja lisa, ojos fondos, bermejos: poco e mal devisa; mayor es que de osa la patada do pisa; las orejas mayores que de añal burrico, el su pescueço negro, ancho, velloso, chico, las narizes muy gordas, luengas, de çarapico: bevería en pocos días caudal de buhón rico.

Su boca de alana e los rostros muy gordos, dientes anchos e luengos, asnudos e moxmordos, las sobreçejas anchas e más negras que tordos [...]

(cs. 1.012-1.014).

Íñigo López de Mendoza, primer marqués de Santillana, fue también un inquieto viajero y, aunque no dejó memoria escrita de sus andanzas, algunos de sus poemas podríamos entenderlos como poéticos relatos viajeros. Nacido en 1398 en Carrión de los Condes (Palencia), don Íñigo pertenecía a la familia de los Mendoza, de antiguo señorío en Álava y afincada desde hacía casi un siglo en tierras alcarreñas de Hita y Buitrago. Por el lado materno, heredaba también un rico señorío en las Asturias de Santillana, comarca a la que hubo de acudir con frecuencia en defensa de sus intereses patrimoniales. Viajó mucho a la Corona de Aragón, asistiendo como copero a Alfonso V, y en 1414 acompañó al infante don Fernando de Antequera en su coronación como rey de Aragón. En la corte catalano-aragonesa, cuyas principales ciudades recorrería, conoció a muchos personajes con los que mantendría trato literario o los celebraría en sus poemas (Juana de Urgel, Violante de Prades, la reina Margarita de Prades, Jordi de Sant Jordi o Enrique de Villena). En 1428 viaja a Portugal acompañando a doña Leonor de Aragón en su casamiento con el príncipe Eduardo. $\mathrm{Al}$ año siguiente es frontero en Ágreda en defensa de los límites de Castilla frente a la incursión de navarros y aragoneses. Realiza un nuevo viaje a Cantabria, a sus tierras del señorío de la Vega, para apaciguar las convulsiones por los pleitos familiares. Pocos meses más tarde se encuentra camino de Andalucía al servicio de Juan II que ha reemprendido la guerra de Granada, aunque tiene que abandonar enfermo en Córdoba. De ahí se retirará a sus posesiones de Guadalajara, Hita y Buitrago, donde permanecerá los años siguientes, recibe a los reyes, organiza festejos y justas, y escribe sus obras más importantes, como la Comedieta de Ponza o los Proverbios.

En 1438 retorna a la guerra de Granada con el cargo de capitán mayor y conquistará victorioso la villa de Huelma y la fortaleza de Bexis. Tras este viaje a Andalucía, firmadas treguas con el rey de Granada en 1439, don Íñigo regresa a Castilla y se apartará de nuevo a sus posesiones de Guadalajara. En 1440 acude al recibimiento de Blanca de Navarra que va a casar con Enrique IV. En 1445 participa en la batalla de Olmedo contra los infantes de Aragón y recibe el título de marqués de 
Santillana y conde del Real de Manzanares. En 1447 asiste a las bodas de Juan II con su segunda mujer Isabel de Portugal en Madrigal de las Altas Torres. Luego de intervenir en algunos hechos de armas por mandato del rey, como en una nueva expedición a la frontera con Aragón y tras pasar un tiempo en su retiro de Guadalajara, Santillana se aliaba con los nobles descontentos para derribar a don Álvaro de Luna, que es ejecutado en la plaza de Valladolid en 1452. A la muerte de Juan II en 1454, se pone al servicio de Enrique IV, a quien acompaña con su ejército en una nueva expedición a la vega de Granada, pero el rey abandona la empresa y se retira a Córdoba para contraer matrimonio con la princesa Juana de Portugal. Don Íñigo marchó a Sevilla y de allí partió en romería al monasterio de Guadalupe. Visitó el santuario y compuso a la Virgen unas coplas devotas, en las que canta los méritos de María e implora su misericordia. Sus últimos años los pasará en el palacio de Guadalajara, donde moría en 1458 (Pérez Priego, 2000).

A pesar de esa extraordinaria movilidad, Santillana no fue un viajero de oficio ni dejó escrito ningún libro de viajes. De alguno de esos viajes sí pudo surgir la escritura, como es el caso del Decir contra los aragoneses de su etapa de frontero, o el de la canción de loores a Blanca de Navarra en su recibimiento, o el de la canción a Isabel de Portugal en su casamiento, o el de las Coplas a Nuestra Señora de Guadalupe. Son estas poesías ocasionales, de circunstancias y de celebración, que no se refieren al viaje en sí.

Pero hay también en la producción de nuestro autor una serie de poemas que sí refieren episodios viajeros, que forman un grupo homogéneo y que, en conjunto, revelarían también características del relato de viajes. Tal es el conjunto de poemas al que los cancioneros dan el título genérico de «serranillas»o «serranas» y a los que, para su mejor identificación, la crítica y los editores modernos han asignado títulos individuales: La serrana de Boxmediano (I), La vaquera de Morana (II), Yllana, la serrana de Loçoyuela (III), La serrana de Bores (IV), Menga de Mançanares (V), La moça de Bedmar (VI), La vaquera de la Finojosa (VII), La moça lepuzcana (VIII), La serrana de Navafría (IX) y La vaquera de Verçosa (X) (Stegagno Picchio, 1966; Marino, 1987).

Todas ellas, en efecto, guardan relación con andanzas viajeras de nuestro poeta: la I y la II tienen por escenario las sierras del Moncayo y se corresponderían con su viaje y campaña militar en la frontera de Aragón en 1429; la III se encuadra en uno de sus viajes a Buitrago, seguramente al regreso del Moncayo a fines de 1429 o principios de 1430; la IV, ambientada en la comarca santanderina de la Liébana, surgiría del viaje que emprende en 1430 a aquellos valles patrimoniales; la V evocaría una de sus estancias en el Real de Manzanares, al pie de la sierra de Guadarrama, entre 1430 y 1438; la VI se localiza en la provincia de Jaén, tierras que recorrió en 1438 durante su campaña de Huelma y Bexis; la VII está ambientada en la sierra de Córdoba, que pudo recorrer también por entonces; la VIII se localiza en la comarca de 
la Llanada Alavesa, que atravesaría en su viaje a la frontera de Navarra para recibir a la princesa doña Blanca en 1440. Aparte de estas ocho composiciones individuales, colaboró también en dos serranillas colectivas, una, La serrana de Navafría, con Rodrigo Manrique, comendador de Segura, y Pedro García de Herrera, señor de Pedraza (IX), y la otra, La vaquera de Verçosa, con Gómez Carrillo de Acuña (X), probablemente ambas compuestas con ocasión del paso de estos caballeros por tierras de Buitrago, asistentes a las justas mantenidas por don Íñigo en Madrid o Valladolid en 1433 y 1434 (Lapesa, 1983).

En el conjunto, podemos apreciar el itinerario que va siguiendo el poeta: se inicia en las sierras aragonesas del Moncayo y el Somontano, continúa por la sierra norte de Madrid en el señorío de Buitrago, sube a la comarca santanderina de la Liébana, retorna a sus tierras de señorío de Manzanares el Real al pie del Guadarrama, de ahí pasa a Sierra Mágina en Jaén y a la sierra de Córdoba, y concluye en el valle de la Llanada Alavesa. Fuera de ese itinerario quedarían las dos serranillas en colaboración, aunque localizadas en tierras del señorío de Buitrago. El itinerario está conformado por el recorrido y estancia en ocho lugares geográficos diferentes, que son las jornadas del Moncayo y del Somontano, la de Buitrago, la de los valles de Liébana, la de Manzanares, la de la sierra de Jaén, la de la sierra de Córdoba y la de tierras de Álava.

Aunque no son etapas continuadas sino separadas en la distancia y el tiempo, hay una intención manifiesta de dar noticia y testimonio de aquellos lugares, de verificar su existencia real. Para ello se recurre a resaltar profusamente los topónimos y nombres de lugar en que transcurre la acción, que no se reducen a uno solo, sino que son varios los que se nombran para marcar mejor los límites geográficos ciertos: Moncayo, Vozmediano, Ágreda, Torrellas, Los Fayos (I); Trasmoz, Beratón, Conejares, Travessaña, Trasobares, Añón, Morana (II); La Gamella, Manzanares, el Boalo, Bustares (V); Torres, Canena, Bedmar, Recena, Valdepurchena (VI). Hay también un particular interés por describir los escenarios, accidentes naturales y elementos paisajísticos del lugar: al pie de «un otero» más «arriba» de Vozmediano (I), arriba «en la montaña» riberas de «una fontana» (II), en los «pinares» del valle de Gamella (V), en un «verde prado» de rosas y flores en la «vía» calatraveña (VII), en el «valle arbolado» entre Gauna y Salvatierra (VIII). Y son prácticamente obligadas las referencias temporales: ya se passava «el verano» (I), después «desta semana» (II), como «esta mañana» (III), ¿quién vos trae «de mañana»? (V), «esta mañana» han corrido la ribera (VI), un día «desta semana», el «albor» del día (VIII), «madrugando» en Robredillo (X) (González López, 1951-1952; Terrero, 1950; Toledano, 1928).

Aunque todos estos relatos se situarían en un arco temporal de 1429 a 1438, conforme a las fechas de los viajes que conocemos, no es seguro que se fueran escribiendo al compás de los acontecimientos. Cabe pensar que se escribieran 
tardíamente, en una especie de ejercicios de estilo, difundidos mayoritariamente en reuniones colectivas palaciegas. A ese auditorio cómplice se dirige a veces el poeta («qual nunca vi dama, / nin otra, señores», IV; «qual tod' onbre la querría, / non vos digo por hermana», VIII) y hasta comparte con él serranas en colaboración (IX y X). Esa familiar complicidad es la que justificaría también la serranilla de recapitulación (VIII), como cierre del ciclo y del recorrido viajero. En esta, los encarecimientos dirigidos a la Moça lepuzcana se hacen por medio de la referencia a las serranas que antes había cantado el poeta y que bien conocería su auditorio:

\author{
Yo loé las de Mocayo \\ e sus gestos e colores, \\ de lo qual non me retrayo, \\ e la moçuela de Bores; \\ pero tal filosomía \\ en toda la Sumontana \\ cierto non se fallaría, \\ nin fue tan fermosa Yllana [...] \\ De la moça de Bedmar, \\ a fablarvos çiertamente, \\ razón hove de loar \\ su gracia e buen continente $[\ldots]$
}

(1999: 124-125)².

En el viaje es también fundamental el encuentro, el conocimiento de otros. En este caso, el género literario determinaba la condición del otro, que, conforme a las leyes de la pastorela, no podía ser sino una vaquera o pastora con la que tenía el encuentro el caballero viajero (Battesti Pelegrin, 1978; Bass, 1996). Lo único que cabía, y era lo que se abría a la sorpresa y al asombro, era la variedad que se permitía en las figuras del personaje. Y es lo que aprovecha al máximo Santillana. A lo largo de su viaje desfilará una variedad de tipos de pastoras y serranas, con lo cual se romperá la monotonía del conjunto, que se presenta más animado en sus episodios y distinto en sus descubrimientos. El personaje de la serrana encarna, en efecto, una diversidad de figuras, que van de la serrana salteadora que guarda el paso, como la de Boxmediano, la de Morana o la de Manzanares, a la más idealizada al cuidado de su ganado, como la de Lozoyuela, la de Bores o la de la Finojosa. Un tanto agresiva y montaraz se muestra la de Boxmediano, viuda de Mingayo, que se lanza al viajero como rayo y quiere compartir con él su zurrón y pasar el invierno. También la vaquera de Morana, moza lozana, le amenaza con su honda pedrera si se sobrepasa y le advierte de la proximidad de su amante Antón,

$2 \quad$ Las citas de los textos de Santillana se hacen por la edición de Miguel Ángel Pérez Priego (1999). 
el vaquero de Morana, con quien pronto hará bodas. La bella Menga de Manzanares, a la que encuentra cantando, controla todo el valle y solo permite el paso a Pascual de Bustares. Tras exigir regalos, le desafía a la lucha amorosa que, con cierto disimulo y con alguna treta, acepta y consuma el viajero. La temprana belleza de Illana, la adornada serrana de Lozoyuela, despierta el deseo del caballero que, en su requiebro, la idealiza y no la considera villana. Merecedora igualmente de otro estado le resulta la hermosa mozuela de Bores («la vuestra beldad / saldrá desd'agora / dentr'estos alcores, / pues meresçe fama / de grandes loores»), que también despierta sus deseos amorosos. Maravilloso e idealizado es el encuentro con la vaquera de la Finojosa. En un marco geográfico fragoso (en tierra de frontera y de tránsito) y un paisaje embellecido (un verde prado de rosas y flores), resplandece la presencia de la vaquera que con su gracia y hermosura desmiente su condición rústica («que apenas creyera / que fuese vaquera / de la Finojosa») y elegantemente rechaza al ocasional pretendiente («que ya bien entiendo / lo que demandades: / non es desseosa / de amar, nin lo espera, / aquessa vaquera / de la Finojosa»).

Como vemos, Santillana va descubriendo en sus encuentros personajes diversos en su presencia, apostura y belleza, pero también en sus maneras y comportamiento. Sin salir de su condición, todas le ofrecen un nuevo y distinto descubrimiento, un nuevo aprendizaje. Como Juan Ruiz, descubre las enseñanzas y diversidad del viaje serrano, y como él frecuenta los mismos parajes de la sierra de Guadarrama y del norte de Madrid. 


\section{BIBLIOGRAFÍA}

BAss, Laura R. (1996). «Crossing borders: Gender, Geography and Class Relations in the Serranillas of the Marqués de Santillana». La Corónica, 25: 1, pp. 69-84.

Battesti Pelegrin, Jeanne (1978). «Tipología del encuentro en la serranilla medieval». En Mélanges à la mémoire d'André Joucla-Ruau. Aix-en-Provence: Université de Provence, t. 1, pp. 405-442.

Calleja Guijarro, Tomás (2004). «Las dos rutas segovianas del Libro de buen amor». En Bienvenido Morros y Francisco Toro (eds.), Juan Ruiz, Arcipreste de Hita, y el Libro de buen amor: actas del Congreso Internacional del Centro para la Edición de los Clásicos Españoles (Alcalá la Real, 9-11 de mayo del 2002). Alcalá la Real: Ayuntamiento de Alcalá la Real, pp. 317-326.

Deyermond, Alan (1970). «Some Aspects of Parody in the Libro de buen amor». En G. B. Gybbon-Monypenny (ed.), «Libro de buen amor» Studies. London: Tamesis Book, pp. 53-77.

Di Stefano, Giuseppe (2001). «Los encuentros serranos y sus relatos en el Libro de buen amor o del arte de la variación». Anuario de Letras, 39, pp. 451-474.

Gernet, Folke (2009). Parodia y «contrafacta» en la literatura románica medieval y renacentista. Historia, teoría y textos. San Millán de la Cogolla: Cilengua.

Gybbon-Monypenny, G. B. (ed.) (1970). «Libro de buen amor» Studies. London: Tamesis Books.

GonZÁLEZ LóPEZ, L. (1951-1952). «Paisaje y pastores en las Serranillas del Marqués de Santillana». Paisaje, 79, pp. 275-278.

KIRBY, Steven D. (1986). «Juan Ruiz's Serranas: The Archiprest-Pilgrim and Medieval Wild Women». En John S. Miletich (ed.), Hispanic Studies in Honor of A. D. Deyermond: a North American tribute. Madison: Hispanic Seminary of Medieval Studies, pp. 151-169.

Knust, Hermann (ed.) (1879). Capitulo que fabla de los enxenplos e castigos de Teodor, la donsella, en Mittheilungen aus dem Eskurial. Tübingen: Litterarischer Verein, pp. 507-517.

LAPESA, Rafael (1978). «De nuevo sobre las serranillas del Marqués de Santillana». En VV. AA., Libro-Homenaje a Antonio Pérez Gómez. Cieza: La fonte que mana y corre, t. 2, pp. 43-50.

LAPESA, Rafael (1983). «Las Serranillas del Marqués de Santillana». En VV. AA., El comentario de textos, 4. La poesía medieval. Madrid: Castalia, pp. 243-276.

MARINo, Nancy F. (1987). La serranilla española: Notas para su estudio e interpretación. Potomac: Scripta Humanistica.

Miletich, John S. (ed.) (1986). Hispanic Studies in Honor of A. D. Deyermond: a North American tribute. Madison: Hispanic Seminary of Medieval Studies.

Morros, Bienvenido y Francisco Toro (eds.) (2004). Juan Ruiz, Arcipreste de Hita, y el Libro de buen amor: actas del Congreso Internacional del Centro para la Edición de los Clásicos Españoles (Alcalá la Real, 9-11 de mayo del 2002). Alcalá la Real: Ayuntamiento de Alcalá la Real.

Pérez Priego, Miguel Ángel (ed.) (1999). Marqués de Santillana. Poesía lírica. Madrid: Cátedra. 
Pérez Priego, Miguel Ángel (2000). «Vida y escritura en el Marqués de Santillana». En Actas del VIII Congreso Internacional de la Asociación Hispánica de Literatura Medieval. Santander: Consejería de Cultura del Gobierno de Cantabria / Año Jubilar Lebaniego / Asociación Hispánica de Literatura Medieval, t. 1, pp. 81-93.

RiQuer, Martín de (1969). «La cuaresma del Arcipreste de Hita y el problema de la doble redacción del Libro de buen amor». En Mélanges offerts à Rita Lejeune. Gembloux: J. Duculot, t. 1, pp. 511-521.

Ruz, Juan, arcipreste de Hita (1992). Libro de Buen Amor. Alberto Blecua (ed.). Madrid: Cátedra.

Simonatti, Selena (2008). "La journée du clerc amoureux». Horas y Eros en el «Libro de Buen Amor» (c. 372-387). Pisa: Edizioni ETS.

Stegagno Picchio, Luciana (1966). «Per una storia della serrana peninsolare: la serrana di Sintra». Cultura Neolatina, 26, pp. 150-168.

TAte, Robert B. (1970). «Adventures in the Sierra». En G. B. Gybbon-Monypenny (ed.), «Libro de buen amor» Studies. London: Tamesis Books, pp. 219-229.

Terrero, José (1950). «Paisajes y pastoras en las Serranillas del Marqués de Santillana». Cuadernos de Literatura, 7, pp. 169-202.

Toledano, J. (1928). «Las Serranillas del Marqués de Santillana». Síntesis, 4, pp. 67-86.

Recibido: 09/03/2021

Aceptado: 21/04/2021 


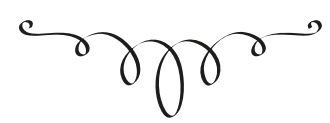

\section{Estrategias DEl RELATO DE VIAJES EN JUAN RUIZ \\ Y EL MARQUÉS DE SANTILLANA}

Resumen: Juan Ruiz, arcipreste de Hita, e Íñigo López de Mendoza, marqués de Santillana, aun en tiempos distintos, residieron en tierras de la cuenca alta del río Manzanares y viajaron por las sierras de Guadarrama y del norte de Madrid. Experiencias vividas en aquellos lugares inspiraron a ambos un buen número de composiciones de alta calidad poética. Examinándolas ahora en su conjunto, podemos descubrir en ellas recursos y estrategias característicos del relato de viajes.

Palabras Clave: Arcipreste de Hita, marqués de Santillana, cantigas de serrana, serranillas, libro de viajes, retrato literario, parodia.

\section{Travel book strategies in Juan Ruiz and el marqués de Santillana}

Abstract: Juan Ruiz, archpriest of Hita, and Íñigo López de Mendoza, Marquis of Santillana, even at different times, lived in the lands of the upper basin of the Manzanares River and traveled through the Guadarrama sierras and north of Madrid. Experiences lived in those places inspired both a good number of compositions of high poetic quality. Examining them now as a whole, we can discover in them resources and strategies characteristic of the travel book.

KEYwords: Archpriest of Hita, Marquis of Santillana, cantigas de serrana, serranillas, travel book, literary portrait, parody. 